Gdańsk 2019, Nr. 40

https://doi.org/10.26881/sgg.2019.40.12

Andrea Rudolph

Universität Opole / Uniwersytet Opolski

https://orcid.org/0000-0001-7576-7087

\title{
Realistische Abrechnung mit Präexistenz und Reinkarnation. Julius von Heydens Gesprächsnovelle Das Geheimnis der Reminiszenz
}

\begin{abstract}
Der Beitrag behandelt eine bisher kaum wissenschaftlich betrachtete Gesprächsnovelle aus der ersten Hälfte des 19. Jahrhunderts. Diese greift den Reinkarnationsglauben als ein Element des deutschen Idealismus zugleich mit liberalen Egalitäts-Ideen auf, um kritisch mit beiden abzurechnen.
\end{abstract}

Schlüsselwörter: Julius von Heyden, Gesprächsnovelle, Friedrich Schiller (Rezeption), Seelenpräexistenz (Kritik), Metempsychose (Kritik), liberale Egalitätsideen

The realistic settlement with pre-existence and reincarnation. Julius von Heydens Conversation-Novella Das Geheimnis der Reminiszenz. Heyden's story takes up the belief in reincarnation as an element of German idealism, as well as liberal ideas of social equality to critically disprove both of them.

Keywords: Julius von Heyden, conversation novella, Friedrich Schiller (reception), soul pre-existence (criticism), metempsychosis (critique), liberal egalitarian ideas

Julius August Leopold Friedrich von der Heyden, Emerentius Scävola (Pseudonym), wurde am 31. Januar 1786 in Mölschow auf Usedom als viertes Kind des Gutsbesitzers Leopold von Heyden geboren. Er verstarb im März 1867 in Melochwitz im Kreis Militsch in Schlesien (heute Miłochowice), wo er Friederike von Usedom geheiratet hatte. Während der Befreiungskriege schwer verwundet, erhielt er eine Zivilversorgung als Postmeister in Königsberg in der Neumark (heute Chojna). Die Postmeisterstelle bot Zeit zu schriftstellerischer Betätigung mit Gedichten, Erzählungen und teils umfangreichen Romanen, ${ }^{1}$ deren Urheberschaft von Heyden durch sein Pseudonym „Emerentius Scävola“ erfolgreich verbergen konnte. Wegen

\footnotetext{
1 Vgl. Schmidt 1840; 174-175, 231; Roßmann 1823; 404.
} 
seiner schweren Verletzung am Arm ergriff von Heyden dieses Pseudonym. ${ }^{2}$ Zeitgenössische Einschätzungen beschreiben Scävola als einen sich „durch Talente und Eigenthümlichkeit" auszeichnenden Schriftsteller (Conversations-Lexikon 1840: 803), rügen aber, er habe sich nach seiner Verwundung und weiteren trüben eigenen und beobachteten Erfahrungen „besonders in Bezug auf die Liebe und das weibliche Geschlecht überhaupt“ eine „dunkle Florbrille“ vor die Augen gesetzt (ebd.: 803). Der Personeneintrag im Conversations-Lexikon verteidigt Scävola umfänglich gegen den von Wolfgang Menzel $^{3}$ und anderen erhobenen Vorwurf, er beschreite den Weg, die „unmoralische Richtung der Neufranzosen" (ebd.) in seinem Werk nachzuahmen und von jenen abzuschreiben. Daneben finde sich auch Krankhaft-Fieberhaftes, ästhetisch und moralisch Ungesundes und Abnormes, Erbsünde, Ehebruch, ja sogar Blutschande in seinen Werken. Dennoch sei Scävola "ganz deutsch, d. h. ehrlich“ (ebd.). Er glaube „an die Wahrheit seiner Figuren“, er wolle "nicht verführen, nicht reizen, nicht pikant sein, nicht erschlaffte Gemüther unterhalten und anstacheln; im Gegentheil“. Er verfolge „eine durchaus ethische Richtung“; seine Personen gingen „oft auf die scheußlichste Weise unter, aber nur, wenn sie es durch Verbrechen und Lasterhaftigkeit verdient haben“ (ebd.). Er wolle „ein Warn- und Schreckbild erstellen“, was ihm „nur zu vollständigem Grade gelungen“ sei. Er stelle „die Sünde, das Laster, die Wollust in absoluter Nacktheit hin“, aber das „moralisch Schlechte in seiner Blöße" reize nicht, sondern „schrecke nur" (ebd.). Während die zu Beginn seiner Laufbahn im „Freimütigen“ erschienenen kleinen Erzählungen „zu den besten“ gehörten, „allerdings schon in der schwarzen Kunst“ gehalten, doch „ziemlich gemäßigt und von großer psychologischer Wirkung “, zeigten die CameraobscuraBilder (1832; neue Auflage 1836) und die Sammlung von Novellen Genossen der Mitternacht (1832) eine „wahrhafte Borussomanie“, einen „blind[en] Haß gegen alles Außerpreußische, eine gewisse körperliche und krankhafte Antipathie gegen Alles, was constitutionelle Staatsverfassung heißt oder danach aussieht“" (vgl. ebd.: 805).

\footnotetext{
2 Man denke an die Scaevola-Geschichte: Die Römer hatten die Etrurier zum Gegner. Als während der Belagerung die Not immer größer ward, schlich sich ein junger Römer, Gaius Mucius, in das feindliche Lager. Er wurde gefangen genommen und verhört. Man drohte, ihn mit Feuer foltern zu lassen. Daraufhin legte Mucius seine rechte Hand ins Feuer und ließ sie verbrennen. Er schwor dabei, daß jeder Römer wie er bereit sei sich zu opfern, um den König zu töten. Dieser schenkte daraufhin allen Römern die Freiheit, Mucius erhielt den Beinamen Scaevola, „der Linkshänder“.

3 Wolfgang Menzel nannte ihn ein „Schwein, welches sich mit einem Battisttuch die Tränen abtrocknet“ (Goedeke 1966; 346). Karl Gutzkow äußerte andere Vorbehalte: Scävola sei „ein Autor der Idee, Leidenschaft und Kunst, aber jedes in einem depotenzirten Grade“. Bei ihm gehe die Idee „nur bis zu der Linie des Sonderbaren und Auffallenden; die Leidenschaft ist ohne Feuer und Subjektivität und Jugend [...]. Die Idee ist hier eine kalte Conception von Außen im Interesse der Neuheit, die Leidenschaft ergreift den Autor nicht selbst, sie bleibt immer nur bald die Wirkung, bald die Ursache seiner Combinationen, und endlich ist die Kunst etwas profan [...]“ (Gutzkow 1839: 348).
} 
Schon der Novellentitel hält einen Fingerzeig für die Inangriffnahme einer Interpretation bereit. Er nimmt populär gewordenes Wissen auf, ${ }^{4}$ von dem das einbezogene Gedicht Schillers lediglich ein Ausschnitt ist. ${ }^{5}$

Ich werde diesen Ausschnitt in einem ersten Schritt zunächst weiten. Ich beschränke mich darauf, den Reinkarnationsglauben als Konstante der Literatur des deutschen Idealismus mit Beispielen zu belegen und zu markieren, dass dessen Konjunktur in der zweiten Hälfte des 18. Jahrhunderts durch aufklärerische Bedürfnisse bestimmt ist. Anschließend wendet sich die Analyse dem gesellschaftsorientierten Erzähltext zu. Ich möchte zeigen, dass diese Gesprächsnovelle von Heydens mit dem idealistischen Reinkarnationsglauben als einem Phantastikon hart abrechnet.

Zunächst: Als die Idee der Reinkarnation das Christentum und die Kirchengeschichte auf ihrem Weg ins europäische Geistesleben verließ, gab es nicht nur einen Umbau, sondern auch einen Kampf um die richtigen Begriffe und Reinkarnationsräume. Wenn Literaten in der zweiten Hälfte des 18. Jahrhunderts ihre Blicke auf das ,alte System“ der Reinkarnation richteten, wurzelte dessen Konjunktur in kulturellen und ideengeschichtlichen Entwicklungen des Jahrhunderts. Die Vorstellung, dass die Seele des Menschen vom Himmel kommend zur Erde niedersteige und von dort wieder emporklimme, assimilierte sich dem westlich-idealistischen Fortschrittsparadigma. Sie bot dabei auch Möglichkeiten, Rückschläge des Einzelnen bei der Ausbildung seiner individuellen Anlagen oder der menschlichen Gattung im Geschichtsprozess - Lessing sprach von Seitwärtsentwicklungen - interpretatorisch abzufangen. Die Auffassung von Kultur als tugendhafter Qualität, um die der Mensch sich bemühen müsse und die ihn Anstrengung koste, ließ sich an Stadienmodelle mühelos anschließen. So verband sich der alte Präexistenz- oder Reinkarnationsglaube mit der idealistischen Kulturierung des Menschen, aber auch mit dem idealistischen Wunsch nach Selbsterweiterung, nach Repräsentanz der Gattung.

Nicht zufällig hatte Lessing in seiner Erziehung des Menschengeschlechts den Reinkarnationsglauben in Verbindung mit theologisch-philosophischen Fragestellungen mit den Mitteln der Vernunft gedeutet (Obst 2009: 126). In seinem 1773 gedruckten Buch Philosophische Gespräche über die unmittelbare Bekanntmachung der Religion und über einige unzugängliche Beweisarten derselben stellte Lessing die Fragen:

Ist es schon ausgemacht, dass meine Seele nur einmal Mensch ist? Ist es denn schlechterdings so ganz unsinnig, dass ich auf meinem Wege der Vervollkommnung wohl durch mehr als eine Hülle der Menschheit durchmüsste? Vielleicht war auf der Wanderung der Seele durch verschiedene menschliche Körper,

4 Die unter Berufung auf Marianne Wünsch formulierte These ist mit Blick auf Heydens Novelle wohl zu relativieren: „Die Idee einer Seelenwanderung, also einer wörtlichen physischen Wiedergeburt, war zwar in der Goethezeit vorhanden, jedoch stand hier primär der Gedanke an verschiedene Zustände innerhalb eines biologischen Lebens, Goethes so genannte ,neue Leben', im Vordergrund. Erst in der Frühen Moderne taucht das Konzept der Reinkarnation häufiger und expliziter auf" (Marko 2006: 8).

5 Reminiszenz heißt: Zurück-Erinnerung. Dese Idee geht auf die Idee Platons von der Anamnese zurück, konkret auf die Erinnerung der Seele an die geistige Existenz vor der Verbindung mit ihrem Körper. Plato hatte in seinem Symposion den Mythos einer vorzeitlichen Einheit des Menschen in männlich-weiblicher Ganzheit erzählt, nach welcher beide Geschlechter sich zurücksehnten. 
ein ganz neues eigenes System zum Grunde? Vielleicht war dieses System kein andres, als das ganz älteste -). (Danzel 1853: 447)

Und im Rahmen seiner kritischen Arbeit über die Philosophische Palingenesie des Schweizer Naturforschers und Philosophen Charles Bonnet (1720-1793) ist Carl Gotthelf Lessings Notiz überliefert:

Dieses mein System ist gewiß das älteste aller philosophischen Systeme. Denn es ist eigentlich nichts als das System der von der Seelenpräexistenz und Metempsychose, welches nicht allein schon Pythagoras und Plato, sondern auch vor ihnen Aegyptier und Chaldäer und Perser, kurz alle Weisen des Orients, gedacht haben. Und schon dieses muss ein gutes Vorurtheil dafür wirken. Die erste und älteste Meinung ist in spekulativen Dingen immer die wahrscheinlichste, weil der gesunde Menschenverstand sofort darauf verfiel. (Lessing 1795: 77)

Zeitgleich hatten auch Mitglieder der Helvetischen Gesellschaft, der badische Hof- und Regierungsrat und Ehemann von Goethes Schwester Cornelia, Johann Georg Schlosser, der Schriftsteller Johann Heinrich Merck und der Pfarrer und Physiognom Johann Kaspar Lavater Gespräche über die Seelenwanderung geführt. Lavater hatte 1769 Charles Bonnets Werk unter dem Titel Philosophische Palingenesie oder Gedanken über den vergangenen und künftigen Zustand lebender Wesen ins Deutsche übersetzt, Lessings Erziehungsschrift von 1781 revitalisierte sicher die Diskussion. Schon zuvor hatte Schlosser erkannt, „dass die kirchliche Lehre in ihrer herkömmlichen Form weder die Frage der Gerechtigkeit Gottes angesichts des Elends und der Ungerechtigkeit in der Welt noch das Problem der ewigen Verdammnis überzeugend beantworten kann und außerdem dem überall zu beobachtenden Entwicklungs- und Fortschrittsprinzip keinen Raum gibt“ (Obst 2011: 129). Im Unterschied hierzu löse die Seelenwanderung diese Probleme ,auf eine, der menschlichen Natur gemäßere Art" (ebd.: 129). Auch neutralisiert er den gängigen Einwand, Christentum und Seelenwanderung schlössen sich aus. Man müsse erst in mehreren Erdenleben reif werden, um den Glauben an Christus und die durch ihn geschehene Offenbarung und Erlösung anzunehmen. Auch in Weimar wurde das Thema viel besprochen. Im Januar 1782 erscheint im „Teutschen Merkur" eine Abhandlung des Generalsuperintendenten Herder Über die Seelenwanderung. Drei Gespräche. Und in seinen Ideen zur Philosophie der Geschichte der Menschheit hatte Herder Kulturen als historisch wandelbare beschrieben, sie im Rahmen einer relativ einheitlichen Lebensweise eingegrenzt auf verschiedene Lebensweisen, Völker und Nationen und die verschiedenen Stadien des Fortschritts nicht nur vertikal, sondern auch horizontal vergegenwärtigt. ${ }^{7}$ Die Gradation wurde im Rahmen der Fortschrittskonzeption zu einem Zentralbegriff der europäischen Aufklärung. Charlotte Buff, die Frau von Goethes Freund Christian Kestner, redete sich mit dem Glauben an die Möglichkeit wiederholten Erdenlebens sicher auch über persönliche Krisen hinweg. Kester notierte am 10. September 1772 in sein Tagebuch: „Er, Lottchen und ich hatten ein merkwürdiges Gespräch von dem Zustande

6 Die Forschung führt den jähen Abbruch der Äußerung unter Berufung auf Lessings Brief an Campe im Dezember 1799 auf eine Erkrankung zurück, die Lessing am Weiterschreiben gehindert habe (Danzel 1853: 123).

„Auch der Kalifornier und Feuerländer lernte Bogen und Pfeile machen und sie gebrauchen; er hat Sprache und Begriffe, Übungen und Künste [...]. Der Unterschied zwischen nicht aufgeklärten, zwischen kultivierten und unkultivierten Völkern ist also nicht spezifisch, sondern nur gradweise“ (Herder 1966: 227). 
nach diesem Leben, vom Weggehen und Wiederkommen etc. etc., welches nicht er, sondern Lottchen anfing" (Bäumer 1919: 188). Die Vorstellung, dass die Seele ein weiteres Mal in einen Leib tritt, bestimmte auch Goethes Deutung seines Liebesaufschwungs, den die ältere und von Zeitgenossen als schön beschriebene Mutter mehrerer Kinder, Charlotte v. Stein, in ihm auslöste. Die metaphysische Behauptung, mit dem Seelenpartner ursprünglich ein Wesen gewesen zu sein, legitimierte Liebeswünsche:

Ich kann mir die Bedeutsamkeit - die Macht, die diese Frau über mich hat nicht anders erklären als durch Seelenwanderung. - Ja, wir waren einst Mann und Weib - Nun wissen wir von uns - verhüllt in Geisterduft. Ich habe keinen Namen für uns - die Vergangenheit - die Zukunft - das All,

schrieb Goethe etwa Mitte April 1778 an Christian Martin Wieland (Maurer 1997: 55). Er suchte eine gemeinsame Erinnerung an vorige Zustände herzustellen, um die Hingabe der sich zurückhaltenden Frau zu erlangen, so auch 1776 in seinem Gelegenheitsgedicht:

Sag, was will das Schicksal uns bereiten?/ Sag', wie band es uns so rein genau?/ Ach, du warst in abgelebten Zeiten/ Meine Schwester oder meine Frau. [...] Und von allem dem schwebt ein Erinnern/ Nur noch um das ungewisse Herz./ Fühlt die alte Wahrheit ewig gleich im Innern,/ Und der neue Zustand wird ihm Schmerz. (Goethe 1981: 123)

Friedrich Schlegel sah die Reinkarnationsmetaphysik ${ }^{8}$ in seinen Vorlesungen bereits in breite Kreise getragen. Acht Jahre nach dem Erscheinen der Novelle hatte er eine Inflationierung des Reminiszenzglaubens in seinen Vorlesungen über die Philosophie des Lebens (1827) beklagt:

In der letztvergangenen Zeit hat man die Lehre von der Unsterblichkeit der Seele - da doch die eigentliche indische Seelenwanderung, so wie wir sie jetzt genau aus den Quellen kennen, zu ernsthaft und zu traurig ist, ${ }^{9}$ um bei unserem Zeitalter viel Beifall und Glauben zu finden - statt dessen nun ganz ins

8 In seinen Vorlesungen über die „Philosophie des Lebens“, die er in den Jahren 1804-06 an der Universität Köln und 1827 weiter entwickelt in Wien hielt, sprach F. Schlegel über drei Unsterblichkeitsvorstellungen. Abzulehnen sei dabei diejenige, die Spinoza (unter dem Einfluss des Buddhismus) vertreten habe: daß die Seele sich nach dem Tode unter Verlust ihrer Individualität wieder in das Einssein des Kosmos auflöse. Ebenso abzulehnen sei jedoch auch die entgegengesetzte Anschauung: dass die Seele nach diesem einen Menschenleben eine ewige Existenz entweder in himmlischem Genuss oder in unendlicher Höllenstrafe erwarte. Die Philosophie habe vorzüglich zwei Grundirrtümer zu widerlegen, „erstens, daß die menschliche Seele sich in nichts aufösen könne, und zweitens, daß die Unsterblichkeit dem Menschen ohne sein Zuthun schon völlig gegeben sey [...]“ [Zit. nach Windischmann 1846: 205)]. Schlegel geht weiter: „Die dritte Meinung von der Seelenwanderung, die Ansicht des Mystizismus, schon wegen ihres hohen Alters so bemerkenswert, liegt in der Mitte der beiden. Diese läßt die Seele nicht gleich, sondern erst nachdem sie mancherlei Formen gewechselt, in die volle Freiheit übergehen. Wir nehmen hier die Seelenwanderung im allgemeinen Sinne als Fortdauer des Geistes bei abwechselnden Formen und Organen" (Windischmann 1846: 203).

9 In den ältesten Texten des indischen Hinduismus, den Veden, erscheint die Idee der Reinkarnation noch nicht. In den Upanishaden, deren älteste auf die Zeit von etwa 800 bis 600 v. Chr. datiert werden, ist sie jedoch ein wesentliches, vielfach variiertes Thema. (vgl. Zander 1999: 25, 31-34). Anders als das Christentum oder der Islam ist die indische Religion nicht von einer bestimmten Persönlichkeit gestiftet worden. Das Band, das diese Religion zusammenhält, ist nicht Wort oder Gedanke eines Stifters, sondern die Kontinuität einer Entwicklung. Nach indischem Glauben ist das Dasein oder Schicksal eines jeden Einzelwesens notwendige Folge seiner Taten, welche es in seinem früheren Leben vollbrachte. „Wie einer handelt, wie einer wandelt, ein solcher wird er: Wer gut handelt, der wird Gutes, wer Böse handelt, etwas Böses“, lehrt eine Upanishad (Glasenapp 1963: 23). 
Romantische hinüberzuziehen und jenes Leben als eine Art von astronomischem Spazierenfahren von einem Sterne zum andern mit lebhaften Farben zu schildern gesucht. (Schlegel 1847: 157)

Bei Schlegel entlud sich im Rückblick auf das Mode-Interesse Überdruss. Dieses sah sich nicht indischen Quellen verpflichtet. Es begnüge sich mit Gemeinplätzen und fröne der von Schlegel abgelehnten Vorliebe für die planetarische Erweiterung des Unsterblichkeitsglaubens. ${ }^{10}$

Ich ziehe aus diesen Belegen folgendes Fazit:

Aufklärungs- und goethezeitliche Literaten bezogen den alten Reinkarnationsgedanken in ihr westliches, von Evolution und Fortschritt geprägtes Paradima ein (Obst 2009: 126, Cyranka 2005: 37). Das „alte System“ stabilisierte die Stufenleiter selbstbestimmten sittlichen Fortschreitens. Dabei verhalf der Glaube an die Möglichkeit wiederholten Erdenlebens dazu, einen starren Schematismus der Hinaufentwicklung zu vermeiden, sich über „tausend Räthsel und Geheimnisse der moralischen Welt" (Schlosser 1781:15) zu beruhigen, mithin gebrochene Schwünge des Verlaufs anzunehmen, und Geschlechterliebe als göttlich zu legitimieren, zuweilen in einer Sprache, die -mit gesuchten Mitteln überredet, überspannt, überrennt. ${ }^{11} \mathrm{Zu}$ den Hypostasen des Vergangenheitsbildes gesellte sich in solchen Fällen eine neue ästhetisch-psychologische Anwendung der Einheitslehre. Einflussreiche Literaten wie z. B. Herder vertraten anstelle der Reinkarnation die Palingenese, die Neuschöpfung nach Ablauf eines Weltzyklus: Nach einer Katastrophe vollziehe sich die Wiederverseelung auf anderen Himmelskörpern.

Heydens Novelle spiegelt nicht lediglich sein Interesse an pathologischen Erscheinungen wider. Sie ist auch eine Abrechnung mit dem Idealismus, der im Banne des Reinkarnationsglaubens stand, ${ }^{12}$ und mit liberalen Zeit-Ideen. In ihrem Erzählfluss ist Schillers Gedicht Geheimnis der Reminiszenz. An Laura (1781) verankert. Im Mittelpunkt der Novelle stehen der Regierungsrat Löwener, dessen Alltagsvernunft auf bürgerlich-irdische Zustände und Erfordernisse eingerichtet ist, seine Gattin Charlotte, Mutter mehrerer Kinder, und deren Schwester Natalie, beide reinkarnationsbegeistert, später gesellt sich Löweners analphabetischer Diener Johann hinzu. Zunächst löst geselliges Lesen ein Gespräch aus. Nachfolgend werden Rückwirkungen der Lektüre auf Verhaltensweisen und Handlungen aller Beteiligten geschildert. Wir haben es demnach mit einer Gesprächsnovelle zu tun. Diese Novellenform reicht tief in die liberale Öffentlichkeitskultur hinein. ${ }^{13}$ In der Anwendung von dargebotenen

10 Sichtlich missfiel Schlegel eine besondere Form der Reinkarnationsspekulation, die das gesamte Weltall belebte und den Menschen nach ihrem Tod ein Weiterleben auf anderen Himmelskörpern zudachte. Der Schweizer Philosoph Johann Heinrich Lambert hatte 1761 Cosmologische Briefe über die Einrichtung des Weltbaues vorgelegt, dort seine Auffassung von der Bewohnbarkeit aller Himmelskörper begründend: „Ich finde die Erde in Kleinem zu sehr bewohnt, als daß ich nicht eben so viele große Wohnplätze gerne annehmen sollte“ (Lambert 1761: V).

11 Die entfesselte Phantasie wird in Schillers Jugendgedicht Geheimnis der Reminiszenz antiklassisch und verliert sich im Grenzenlos-Überschwänglichen.

12 Hierfür hat (Obst 2009) eine gut dokumentierte Übersicht geboten. Von Lavaters Übersetzung des Buches von Charles Bonnet ins Deutsche: Philosophische Palingenesie oder Gedanken über den vergangenen und künftigen Zustand lebendiger Wesen "gingen im 18. Jahrhundert immer wieder Anregungen für unterschiedliche Seelenwanderungsspekulationen aus" (Obst 2009: 127).

13 Die Abhängigkeit der Gesprächsnovelle von der liberalen Geselligkeitskultur und ihre Beförderung durch dieselbe sind hinreichend bekannt. Vgl. Sengle 1972: 54; Rudolph 2018. 
Urteilen und Meinungen, philosophischen Theorien oder geläufigen Lebensanschauungen suchte dieser auch in Schlesien äußerst produktive Novellentypus Alltagserfahrungen mit anderen Mitteln in politische Fragen einzubinden als seine Schwester, die symbolisierende dramatische Novelle, für die Kleist das Muster abgab.

Fordert Löwener bei Tisch „freundliche Geschichten“, die - ganz im Sinne von Theodor Mundts 1834 in Umlauf gebrachter Metapher von der Novelle als bürgerlichem „deutsche[n] Hausthiere" (Polheim 1970: 71) - einen Bezirk des Friedens schaffen sollen, begegnet ihm diesmal schweigsame „Kopfhängerei“ (Scävola 1832: 2). Schuld ist die Schillerlektüre. Die Schwestern sind bewegt von Schillers Spekulation, wonach unstillbares Liebesverlangen nach einem Du aus früheren Liebeswonnen stammen müsse. Solche Wonnen habe die in einem neuen Körper wiedergeborene Seele mit dem leidenschaftlich begehrten $\mathrm{Du}$, hier mit Laura angesprochen, in ihrem Leben vor dem aktuellen Erdenleben genossen. Auch das geliebte Du habe sich nach dem Tod seines vordem besessenen Körpers wieder inkarniert und Erlebtes in seine Wiederfleischwerdung mitgenommen. ${ }^{14}$ Die Leidenschaft - mit ihrer durchaus physiologischen Symptomatik: Blutfülle und Schwindel, Liebesglühen - erhält so ein Recht, auch wenn nicht um ihrer selbst willen. Integriert in ein heilsgeschichtliches, theosophisches System ist sie mit Vorstellungen vom Göttlichgewesensein und intendiertem Wieder-Göttlich-Werden des Menschen verbunden. Einst waren die Liebenden in einer Welt über den Sternen als „innig festverbundene Wesen“ eins mit Gott: „Uns entgegen gossen Nektarwellen/ Ewig strömend ihre Wollustwellen,/ Mächtig lösten wir der Wahrheit Siegel, / Zu der Wahrheit lichtem Sonnenhügel/ Schwang sich unser Flügel" (Scävola 1832: 5). Bald aber folgte eine Zerstreuung dieser Einheit. Es entstand eine Welt der vielfältigen Erscheinungen, unter den mangelhaften Einzelwesen auch die Menschen als „des Gottes schöne Trümmer“ (ebd.: 1832: 6). Unwillkürliche Erinnerungen führen die defizitären Einzelwesen aus ihrem in die Gegenwart gebannten Dasein ins Vergangene. Erinnerungen drängen zur „Aufhebung der Trennung ${ }^{15}$ durch „Liebe“ (Schiller NA 20: 121, 124) ${ }^{16}$ - so der „metaphysische Roman“, in den Gott, Mensch und Welt eingefasst sind (Riedl 2002: 197). Nicht das teleologische Gerichtetsein der „Prüfungswallfahrt“ (Scävola 1832: 7) auf Gott ist zwischen den Frauen strittig. Strittig ist der Ausgangspunkt. Charlotte bezieht diesen auf ein metaphysisches Lichtwesen, Natalie auf das Tier. Diese Sicht ist jeweils durch eigene Bedürfnisse mitbestimmt.

14 Die petrarkistische Liebesbeschreibung findet sich hier nicht in typologischer Reinheit. Mehr als durch den Leib wirkt die Frau durch die Tugend auf den Dichter, das erotische Verhältnis hat somit sein Schwergewicht im Seelischen. Es muss sich vor allem auf seelische Kommunikation beschränken. Das war die Liebesauffassung des echten Petrarkismus.

15 Siehe: „Weine Laura! Dieser Gott ist nimmer/ Du und ich des Gottes schöne Trümmer, / Und in uns ein unersättlich Dringen, / Das verlorene Wesen einzuschlingen, / Gottheit zu erschwingen“" (Scävola 1832: 6).

16 Riedl verweist auf Schillers baldige Verabschiedung seiner Jugendphilosophie, ausgelöst durch sein Bekanntwerden mit religionskritischen Schriften französischer Materialisten, aber auch David Humes (vgl. Riedl 2002: 198). „Es erfolgt ,die Umschreibung' des theo-historischen Konzepts in ein welt- und zeitimmanentes, wie es exemplarisch in dem universalgeschichtlichem Poem Der Künstler von 1789 vorliegt “ (vgl. ebd.: 199). 
Charlotte meint, als Engel habe der menschliche Geist seine frühere Heimat verlassen, sein Körper zog ihn nieder zur Erde. Nun strebe er erneut zu Gott empor, indem er seine Sinne „in ihre ursprüngliche Sklaverei zurückkämpft“. Natalie hält dagegen: „Nicht als ein Engel“, sondern als „niedre Tierseele “ habe der Mensch seine „frühere Heimath“ verlassen, „als niedre Thierseele zerbrach er das ihm zu enge gewordene Gehäuse, um - mit vervollkommneten Sinnwerkzeugen ausgestattet - seiner Vollendung entgegen zu reifen" (Scävola 1832: 9). Dabei werde der Schmerz als ,rauer Mitkämpfer“ hilfreich sein, denn er reiße den Menschen „hinweg von den verlockenden Freuden“ (ebd.: 11). Die Seelen, die während ihrer Erdenschicksale wachsen und reifen, steigen sukzessive in Himmelsebenen auf. Die anderen, die ihre Dämonen nicht niederringen können, werden in längst verlassene Tiefen zurückgeschleudert, aus denen sie ,immer wieder von neuem klimmen“ (ebd.: 10).

Der Geheimrat verspottet die Anschauung, wonach Menschen mehrere Erdenleben hinter sich bringen, als "ganz etwas Neues" (ebd.: 3). Sicher fasst dies seinen idealismusfeindlichen Affekt mit einem Ausdruck zusammen. Zudem befindet sich dieser Ausdruck im Einklang mit der Form. Denn hersehend von Goethes berühmter Charakteristik der Novellengattung im Gespräch mit Eckermann am 29. Januar 1827 stehen die Behauptung der Präbzw. Fortexistenz von Seelen in aufeinander folgenden Leben zu bisherigen Erfahrungen tatsächlich im Verhältnis des Unerhörten. Die Frauen setzen sich gegen den Spötter zur Wehr. Natalie stützt das „System“ auf die Evidenz von Lebenserfahrung. Ihre schnell entschlossene Heirat mit ihrem nunmehr bereits verstorbenen Mann führt sie auf „Ahnungen aus einem früheren Leben“ zurück, beider „Wiedererkennen war ein gegenseitiges gewesen“ (ebd.: 12). Löwener stichelt:

Ihr seliger Mann war herzensgut, aber eine Biene mag er wohl früher nicht gewesen sein, denn er verstand das Einsammeln nicht [...]. Eine Schnecke ist er auch nicht gewesen, denn er war nicht gewohnt, mit den Fühlhörnern vorsichtig seine Umgebung zu untersuchen, [...]. (Scävola 1832: 18)

Die fortschreitende Handlung unternimmt die Geltungsprüfung geäußerter Ansichten. Löwener tritt eine Reise in sein Ministerium an. ${ }^{17}$ Sein längeres Ausbleiben begründet er in einem Brief. Er habe Natalies System, wonach „die Seele eines Menschen durch seine Stimme, durch seinen Blick, durch seine Mienen“ spreche, ,auf schwachen Füßen gefunden“ (Scävola 1832: 19). Denn gerade der Diener, der ihn durch sein gefälliges Äußeres eingenommen hatte, bestahl ihn, es sei Zeit verstrichen, bis er wieder eingefangen war. Inzwischen habe er ganz bewusst einen „Gegenfüßler" des Systems (ebd.: 23), einen unsympathisch wirkenden Menschen, in den Dienst genommen. Der zur Widerlegung des Systems Angeworbene wird paradoxerweise in dieses Ordnungssystem integriert, von Charlotte nämlich in die erotisch-partnerbezogene Variante. Beim ersten Anblick schon verfällt sie ihm. Der Diener zieht als Ehemann aus einem früheren Erdenleben an ihrer Seele vorüber: „Aus ihren stieren Augen war alles Licht, alle Sehkraft für das Sichtbare entschwunden; sie sah Nichts als das Bild ihrer jüngsten und, sie gestand es sich mit Beben, ihrer ältesten Reminiszenz" (ebd.: 23). Derart wird ihre Einhüllung in den Reminiszenz-Wahn beschrieben - in Wahrheit

17 Wie im patriarchalischen Familienmodell üblich, verbindet sich mit Charlotte ein auf den Familienkreis bezogenes Dasein, mit Löwener ein nach außen gerichtetes Leben. 
eine Sublimierung sexueller Affektzustände, wie man seit $\mathrm{S}$. Freud sagen würde. Freilich ist die intrapsychische Dynamik der Selbstbeobachtung den Figuren selbst nicht zugänglich. Doch via Erzählkommentar und weiterer Mittel erhält der Leser Hinweise darauf, dass die leidenschaftliche Affektion damit zusammenhängen muss, dass Löweners eheliche Liebe weniger imponierend ausfiel. Den Gleichstellungsenthusiasmus des theosophisch- utopischen „Lehrgebäudes"verknüpft der Erzähler mit Revolutionsideen. Zunächst hebt sich - von der Metaphysik her gesehen - ein Menschenbild auf, das auf äußeren Faktoren wie Herkommen und Standeszugehörigkeit beruht: Dieser Mann „mit der deutlich aus seinem Auge flammenden Seeleninschrift: ,Du bist mein “ konnte nicht der sein, den sich ihr Mann „zu den niedrigsten Dienstverrichtungen verdungen hatte“ (Scävola 1832: 23), sinniert Charlotte. Der Affekt wirft sie auf das Krankenlager. Bald darauf brechen die bürgerlichen Bestimmungen von Familie zusammen. Als der kleine Otto sich in Krämpfen windet, bleibt die sonst liebende Mutter unberührt. Der Kindesvater und Ehemann wird dieser zu einer ganz gleichgültigen, ja feindseligen Person.

Nachdem das Kind inneren Verletzungen erliegt, bezichtigt Johann sich des Mordes. Versehentlich hatte er den unten am Wagen knienden Jungen beim Sprung vom Wagen in den Bauch getreten. Dieses Unglück mit Todesfolge und Charlottes insgeheim geäußertes Flehen, Johann müsse weggeschafft werden, sonst sei sie verloren für ihren Mann, für die Kinder, für alles, was sie bisher ,als Pflicht und Tugend erkannt“(Scävola 1832: 26) habe, nimmt Natalie als Bestätigung ihrer idealistischen Basisannahme. Aber sie rügt die Vermischung von moralischem Entwicklungsglauben mit dem Liebestrieb. Sie mahnt Charlotte zur Besinnung auf die Familienbande, auch um sie für ein asketisches Programm des „Systems“ zu retten, und dringt in den Geheimrat, den Diener zu entfernen: „Dieser Johann war der Feind Ihrer Seele in dem Leben jenseits der Wiege!“ (ebd.: 27). Löwener wirft die Schwägerin aus dem Haus, die seiner Frau das Gehirn verbrannt habe und korrigiert seine Personalentscheidung keineswegs.

Indessen stieg Charlottes Fieberglut. Die verhüllten Äußerungen der psychischen Instanzen zu identifizieren, ist des Arztes „Heilkunst nicht ausreichend“ (ebd.: 29). Während dieser diagnostisch im Dunkeln tappt, zieht die Kranke den erschrockenen Diener mit durstigen Küssen zu sich herab. Als Johann ihr Stammeln vernimmt - „Mein! Mein Seelenvermählter! - Gefährte meines frühern Lebens!“ - hält er sie für „wahnsinnig“ (ebd.: 33). Trotz solcher Sehnsuchtsschübe erkennt die Kranke die Diskrepanz zwischen ihren Erinnerungsbildern und dem Mann, dem in diesem Leben Zucht, Sammlung, die Askese fehlen. Er unterscheidet sich von dem Mann, der er bei seiner letzten Verkörperung war. Sie redet sich ein, sie könne seine verunreinigte Natur zum „Übersinnlichen“ (ebd.: 60) hinleiten. Ihre bei der Prüfungswallfahrt schon weit vorausgeeilte Seele müsse vor dem Ziel stehen bleiben und umkehren zu dem unglücklichen Johann, der sinken musste, um sie zu prüfen. An der Hand ihres Seelenfreundes will sie „,om Leben zu Leben und durch tausendmal tausend Gräber" wallfahren und auf rauer Bahn an seiner „Veredelung" (ebd.: 47) arbeiten. Der Arzt rät eine Gesundungsreise nach Bad Landeck an. Johann, dem Löwener Charlotte, Pferde und Kutsche anvertraut, gelingt es, die Köchin Lisette mitreisen zu lassen. Und er kann Charlotte überreden, die Livree während der Reise ablegen zu dürfen. Sie interpretiert seinen Wunsch, „das schmachvolle Sklavengewand“ abzulegen, als Ausdruck von „Seelenadel“ (ebd.: 59). Reinkarnationsromantik verabschiedet die ständische Schichtung. „Er fing an, 
das Mißverhältniß seiner Stellung zu seinem Berufe zu erkennen, und hatte den ersten großen Schritt auf der Vollendungsbahn gethan" (Scävola 1832: 59).

Ein Zusammenhang ihrer Illusion, er wolle beider uranfängliche Ebenbürtigkeit in Wirklichkeit verwandeln, mit französischen Revolutionsideen ist offenkundig. Dass das Ausziehen des Sklavengewandes Johann in den Sinn kam, hängt freilich allein mit kriminellen Plänen zusammen, die so besser vor einer Entdeckung geschützt erscheinen. Bereits vier Stunden nach Fahrtantritt hatte der Dienstmann seine Livree mit einer ,sehr anständigen bürgerlichen Kleidung" gewechselt. Dass es sein Körper ist, den die Seele für eine bestimmte Anzahl von Jahren bewohnt, der verlockend wirkt, und nicht seine Seele, macht Charlotte sich nicht bewusst. Freilich sieht dies der Erzähler.

Am dritten Tag der Reise wird Johann von einem Landsmann verhöhnt. Aus den bissigen Bemerkungen geht hervor, dass Johann eine Ehefrau hat, die gerade im Kindbett liegt und glaubt, ihr Gatte sei im Krieg gefallen. Die Jahre der napoleonischen Eroberung Deutschlands klingen an. Flüchtend kutschiert Johann die Reisegesellschaft von dannen. Die Geschichte, die Johann Charlotte erzählt - aus der unteren Volksklasse stammend wurde er an die langjährige Geliebte seines jungen Herrn verheiratet und bekam dafür das Amt des Schulmeisters, in dem er, des Schreibens unkundig, tagtäglich dem Hohn der Bauern ausgesetzt war - gehört sicher zum sozialkritischen Realismus des 18. und 19. Jahrhunderts. Als Charlotte nach dieser Erzählung ihren Seelenverwandten zu sich emporzuheben glaubt und ihre Ermahnung, er möge sich scheiden lassen und der jungen Mutter die Hälfte seines Lohns überlassen, durch die Geste aufgehobener Arme unterstreicht, stürzt er sich in dieselben. Dass er sich - um irdischer Güter willen - hineinwarf, verrät schon seine Antwort „am Morgen danach“, er wolle seinen ganzen Lohn für sich behalten. Am selben Morgen hört Charlotte von ihrer aufmüpfig gewordenen Köchin Lisette, die den Sexualakt belauscht hatte, dass Johann dieser die Ehe versprochen hatte. Ihre Eifersucht habe er mit der Erklärung besänftigt, Charlotte sei ihm zu alt, er wolle lediglich ihre Zuneigung benutzen, um ein Herrenleben zu führen. Daran habe er ihr einen Anteil versprochen. In den darauf folgenden Tagen erlebt die Gattin des Geheimrats den Schrecken einer Volkserhebung.

Lisette fuhr fort, mit der ganzen Frechheit eines, zur Herrschaft emporgestiegenen Sklaven, ihre Geißel gleich dem Schwert eines Eroberers, über das Haupt ihrer gestürzten Gebieterin zu schwingen, welche willen- und gedankenlos den eisernen Zug ihrer Züchtigerin auf ihrem gebeugten Nacken duldete (Scävola 1832: 78) -

so die Mechanik des Zwangsläufigen. Es ist offenkundig, dass Lisettes Umkehrung der Sozialordnung als kriminelle Umverteilung erscheint. Bedenkt man das Ausmaß der beruflichen Einbindung Heydens in die Gestaltung des preußischen Staates, überrascht das nicht.

Charlotte sucht der unerträglichen ständegesellschaftlichen Umkehr zu entfliehen, Johann fängt die apathisch wirkende Frau ab, schlägt ihr die gemeinsame Flucht nach Sachsen vor, freilich nicht ohne sie aufzufordern, in den Gasthof zurück zu schleichen und ihre Geldkassette an sich zu nehmen. Als die Köchin am nächsten Morgen noch Charlottes Taschenbuch, deren Reisepass, einiges Papiergeld und einen Staatschuldbrief über 500 Taler findet, reist sie als Regierungsrätin Löwener ins Bad Landeck. Die nach Sachsen Entflohenen gelangen nach Oederan. Dort entdeckt Johann ein Häuschen, „welches seinen Wünschen zusagte“" (Scävola 
1832: 82). Als die Ortsobrigkeit den Ausweis des Kaufinteressenten verlangt, erregt er den Verdacht des Beamten. Als Frau Loewener aufgespürt wird, macht Johann sich samt den Pferden aus dem Staub. Der Gerichtsdiener findet eine augenscheinlich Kranke aus besserem Hause vor. Nachdem ein hinzugezogener Beamter registriert, dass Charlotte „mit fliegendem wahnsinnsverrathenen Blick“ den Flüchtigen ihren „Seelenvermählten“ tituliert (ebd.: 84) und zudem noch über Bares verfügt, wird sie in das nahe liegende Irrenhaus eingewiesen. Nachdem der an der böhmischen Grenze Zurückgewiesene endlich gefasst ist, ordnet der Beamte Bestrafung mit den Worten an: „ein solches Bürschchen wird am besten im Zuchthaus veredelt“"(ebd.: 85). In solchen Worten manifestiert sich, um mit Michel Foucault zu sprechen, ein bestimmter Diskurs. Foucault führt in seiner Schrift Überwachen und Strafen. Die Geburt des Gefängnisses (1975) zum Erfolg der Disziplinierungstechniken der modernen Gesellschaft aus, dass sich die Praxis der Strafgewalt um 1800 zu ändern begann. Es vollzog sich der Schritt weg von den im Feudalismus noch gegen den Körper gerichteten Strafen (Stichwort: Hinrichtungsfestlichkeiten mit direkter Gewaltausübung am Körper) hin zur Humanisierung des Strafvollzugs. Dabei wurde dann auch das Innenleben des Delinquenten entdeckt, was eine ganze Justiz-Pädagogik mit sich brachte. Tatsächlich verleiht von Heyden einer solchen Besserungspädagogik Beweiskraft. Sichtlich bestätigt die Handlung des sterbenden Johann die Effektivität des Zuchthauses bzw. Arbeitshauses als Erziehungsanstalt. Heyden nutzt ein Bild, das das Emporheben der Sinkenden eindringlich widerspiegelt, das nun aber keine metaphysische, sondern realistische, lebensrettende Bedeutung erhält. Auf dem Wege zu Charlotte erfährt Natalie, dass über Waldheim ein starkes Unwetter getobt und der angeschwollene Zschopaufluß die Straßen überflutet hatte. Infolgedessen waren die leichteren Häftlinge des Zuchthauses zur Verlegung des angrenzenden Irrenhauses herangezogen worden. Dabei hatte ein Mann den Tod gefunden. Zuvor hatte der Verunglückte

eine Frau ergriffen, um sie durch die Fluten zu tragen, war aber, vom Wellendrange auf dem inneren Hof umgeworfen, dem Thore zugetrieben, und mit tödlicher Gewalt an einen Pfeiler geschlagen worden. Dennoch hatte er, selbst sterbend, noch so viel Bewusstsein behalten, daß es ihm möglich gewesen war, sich mit der einen Hand an ein Treppengeländer festzuhalten, und mit der anderen die ergriffene Frau über dem Wasser zu halten. (Scävola 1832: 94)

Natalie identifiziert den im Zuchthaus ethisch geretteten Toten als Johann. Im Spital findet sie Charlotte, die ihr ihre Kinder anvertraut, sterbend, aber noch immer an eine postmortale Zukunft glaubend. Von den Begebenheiten mitgenommen, verbringt Natalie einige Wochen mit einer bejahrten Reisebekanntschaft namens Luzie auf einem Landsitz bei Bacherach. Dieser schreibt der Erzähler einen ruhigen, nüchternen Verstand zu, mit dem sie das Geschehen beurteilt, das Natalie ihr anvertraut. Von ihr wird am Ende ein spezifischer Blick auf das Geschehen geworfen. Indem die bejahrte Dame die Besinnung auf die „Wahrscheinlichkeit" einfordert, bekämpft sie das System „sehr gelassen, aber sehr bestimmt“ (ebd.: 99). Sie weist auf den Zusammenhang von Liebesmangel und Empfänglichkeit für diese Art von Metaphysik. Das unerfüllte Eheleben Charlottes war quasi der Boden, auf dem die Bäume romantischer Sehnsucht nach einem Seelenverwandten in den Himmel wuchsen. Sie erinnert Natalie daran, dass ihre Schwester als junge Ehefrau das Bedürfnis hatte, Erwiderung ihrer Zuneigung zu finden. Dass Charlottes Phantasie längst abhängig von ihren Begierden geworden sei, 
habe das Fieber ausgelöst, noch bevor Johann ihr ins Auge fiel. Eine metaphysisch-pädagogische Verklärung sexueller Attraktion zur eingebildeten Pflicht, den Gesunkenen emporzuheben, entlastete Charlotte von der Prosa einer ehebrecherischen Leidenschaft.

Als Natalie zufällig Löwener wieder begegnet, die vornehm aufgeputzte Köchin als Geliebte diesmal an seiner Seite, steht der Geheimrat keineswegs mehr in geharnischter Opposition gegen das System. Wie seine Frau setzte auch er das System an die Stelle der sozialen und kirchlichen Verbundenheit. Auch er erschlich sich auf diese Weise Ablass für seine sinnliche Schwäche. Freilich hält er sich mit idealistisch-pädagogischer Kostümierung weit zurück, wenn er erklärt, er habe schon länger mit der Köchin seine Bedürfnisse ausgelebt, anfänglich mit Gewissensbissen, doch als er auf das System zurückgriff, seien diese verschwunden. Was sich in Charlotte unbewusst vollzog, ihr waren die psychischen Vorgänge verhüllt, ist bei Löwener lebenspraktische Berechnung. Als der Geheimrat bald darauf an einer falsch diagnostizierten Fußverletzung stirbt, er geriet bezeichnenderweise unter die Räder seiner Kutsche, wird er von der Köchin und Geliebten ausgeplündert.

Der Gesprächsnovelle entspricht, dass eine finalistische Lösung unterbleibt. Am Ende heißt es, es sei nicht bekannt, ob Natalies Glaube an das System lebhaft geblieben ist. Aber sicher sei, dass sie diesen nicht an die ihr anvertrauten Kinder ihrer Schwester weiter vermittelte. Dass die Novelle nicht mit einer naiv-finalistischen Anschauung des Richtigen abschließt, lud den Leser dazu ein, sich über sich selber zu verständigen. Die Schwestern unterwarfen ihr Tun ja Ansichten, die andere Figuren und vielleicht nicht wenige Leser teilten. Folgt man der Handlung freilich, präsentiert sich das romantische „System“ als Trug und Existenzvernichter, der Charlotte einen Totalverlust an Besitz und Verstand einbrachte.

Mit dieser Novelle hatte von Heyden Zeittendenzen als individuelles Schicksal gestaltet. Das System, das Natalie und Charlotte so außerordentlich hoch stellten und Löwener sich pragmatisch zunutze machte, führte zur Auflösung des Eheverhältnisses und der Familie, zu Krankheit und Tod. Dazu tritt nun, und nicht am Rande, dass die für die Dienstpersonen hergestellte gesellschaftliche Gleichheit beide Male gegen sie ausschlägt. Zweifelsohne entstand dieser Text nicht nur in einem bestimmten historischen und sozialen Kontext, von Heyden wollte auf diesen auch einwirken. -

Es lassen sich gattungstheoretische, literatursoziologische, literarhistorische und politische Lineaturen herausheben.

1. Die Novelle ist systematisch dem Gespräch zugeordnet, was zur Formeigentümlichkeit der literatursoziologisch interessanten Diskussionsnovelle gehört. Dass diese die Verabschiedung der Präexistenz- und Reinkarnationsmetaphysik intendiert, verweist literarhistorisch auf den Bruch der Realisten mit den idealistischen Systembildungen. ${ }^{18}$ Demythisierung durch Psychologisierung gehörte bald zum Repertoire materialistischer Anthropologie - bis hin zu Sigmund Freud.

2. Die Novelle enthält durchaus einen politischen Sinn. Auf zwei Stufen führt Heyden eine Entgrenzung bürgerlicher Ordnung vor. Zunächst erscheint die Reinkarnationsgläubigkeit als Abhilfsbewegung gegen eine leere Ehe und löst die Familie auf. Dabei verbindet sich der

18 Freilich hat auch der Bruch der Realisten mit den idealistischen Systembildungen die Wirkungsgeschichte des Idealismus keineswegs beendet. 
Idealismus, von dem Lotte phantasiert, mit der sozialen Frage. Der Autor knüpft an idealistische Allgemeinvorstellungen einer Kranken Forderungen der untersten Volksklasse nach Aufhebung sozialer Ungleichverhältnisse. In die idealistische metaphysische Vorzeichnung uranfänglicher und heilsgeschichtlich wiederzugewinnender Gleichheit schreiben sich Forderungen aus dem Revolutionszeitalter ein: Dienstboten fordern die Ablehnung des Sklavengewandes. Nackte Bereicherungsinteressen kleiden sich sprachlich in das Kostüm idealer Forderungen. Zeigt der romantische Utopismus einen anarchischen Charakter, da er die Aufhebung von Familie und ständischer Ordnung zur Konsequenz hat, wird sein a-bürgerlicher Effekt durch die Kontamination mit kriminellen Handlungen (Betrug, Urkundenfälschung, Diebstahl $)^{19}$ noch verstärkt.

3. Dennoch intendiert die Novelle nicht, der Gegenwart jede utopische Dimension abzuschneiden. Zum einen stammt die Romantik, die dem bürgerlichen Leben störend entgegentritt, nicht schlechthin von außen, sondern aus tiefsten menschlichen Schichten und Bedürfnissen. Zum anderen hält ein tragischer Schluss der Wirklichkeit andere Möglichkeiten entgegen. Heydens Text geht zur seelischen Verarmung bürgerlicher Institutionen ebenso wie zu deren Aufösung auf Distanz. Derart erscheint eine Ethisierung bürgerlicher Institutionen als conditio humana.

4. Es stellt sich die Frage nach dem erzählstrukturellen Verfahren. Der Typus „Gesprächsnovelle" besaß die Funktion der Konfrontation philosophischer Thesen und Lebensanschauungen mit Alltagserfahrungen. Das psychologische und soziale Stimmigkeitskriterium gehört zum Realismus ${ }^{20}$ mitsamt einer Erzählerfigur, die die Sequenz der Ereignisse vollständig überschaut. Heydens Novelle schafft nicht mehr synthetisierte Figuren auf der Basis geschichtsphilosophischer Konstruktionen, also romantische Allegorien, für die soziale und psycho-logische Wahrscheinlichkeit gar nicht intendiert war. An die Stelle solcher Entwürfe sind lebendige Charaktere und die Erklärungskraft lebensbiographischer Elemente getreten, etwa agiert Johann in Übereinstimmung mit seiner Vorgeschichte. Die realistische Novelle bedient sich - hier im Fall Charlottes - introspektiver Darstellungsverfahren, um die Innenwelt von Figuren zu erklären. Festzuhalten bleibt, dass der Systemglaube vom Gang der Ereignisse selbst wie von den Mutmaßungen der Freundin und durch den Erzählkommentar relativiert wird.

\section{Literatur}

Bäumer, Gertrud (1919): Goethes Freundinnen: Briefe zu ibrer Charakteristik. Ausgewählt und eingeleitet von Gertrud Bäumer. Aufl. 2. Wiesbaden: Springer Fachmedien.

19 Nicht nur bittet der Dienstbote schon vor der Abreise um Geld, er hatte auf Rechnung seiner Herrschaft auch einen Kredit aufgenommen und fürchtet nun angedrohte Beschwerden bei seinem Herrn.

20 Bei der Verwendung dieser Bezeichnung schließe ich mich dem Arbeitspragmatismus Rainer Rosenbergs an: „Epochenbegriffe können als der Ökonomie des literaturwissenschaftlichen Diskurses dienende Abbreviaturen funktionieren, sofern über Strukturen und Grenzen der semantischen Felder, die sie aufrufen sollen, ein ungefährer Konsens herstellbar ist" (Rosenberg 2013: 86). 
Bobert, Sabine (2009): Postmoderne Präsenz der Mythen - Auferstehung, Reinkarnation, Esoterik. In: Philipp David, Hartmut Rosenau (Hgg.): Auferstehung: Ringvorlesung der Theologischen Fakultät Kiel. Münster: LIT, 189-220.

Conversations-Lexikon der Gegenwart in 5 Bde. 1838-1841. Bd. 4 (1848): P bis S. Leipzig: Brockhaus.

Cyranka, Daniel (2005): Lessing im Reinkarnationsdiskurs: eine Untersuchung von G. E. Lessings Texten zur Seelenwanderung. Göttingen: V \& R unipress.

Danzel, Theodor Wilhelm (1853): Gotthold Ephraim Lessing, sein Leben und seine Werke, Bd. 2. Leipzig: Dyk'sche Buchhandlung.

Glasenapp, Helmuth von (1963): Die fünf Weltreligionen. Hinduismus, Buddhismus, Chinesischer Universismus, Christentum, Islam. München: Diederichs.

Gnasenapp, Helmuth von (1966): Das Indienbild deutscher Denker. 2. Aufl. Stuttgart: Koehler.

Goethe, Johann Wolfgang (1981): Werke. Kommentare und Register. Hamburger Ausgabe in 14 Bde. Bd.1: Gedichte und Epen. Textkritisch durchgesehen und kommentiert von Erich Trunz. München: Deutscher Taschenbuchverlag.

Gutzkow, Karl (1839): Beiträge zur Geschichte der neuesten Literatur. Erster Band. Stuttgart: Balz.

Jacob, Herbert (Hg.) (1866): Grundriss zur Geschichte der Deutschen Dichtung. Aus den Quellen von Karl Goedeke, 2. neu verarbeitete Aufl., Bd. 14. Achtes Buch: Vom Frieden 1813 bis zur französischen Revolution. Hannover: L. Ehlermann.

Lessing, Carl Gotthelf (1775): Gotthold Ephraim Lessings Leben nebst seinem noch übrigen literarischen Nachlasse, Anzeige seiner philosophischen und theologischen Bruchstücke. Berlin: Vossische Buchhandlung.

Maurer, Doris (1997): Charlotte von Stein: eine Biographie. Frankfurt a. M.: Insel.

Michaels, Axel (1981): Wissenschaft als Einheit von Religion, Philosophie und Poesie. Die Indologie als frühromantisches Projekt einer ganzheitlichen Wissenschaft. In: Wilhelm Halbfass (Hg.): Indien und Europa. Perspektiven ihrer geistigen Bewegung. Basel: Schwabe, 325-340.

Obst, Helmut (2009): Reinkarnation. Weltgeschichte einer Idee. München: C. H. Beck.

Polheim, Karl Konrad (Hg.) (1970): Theorie und Kritik der deutschen Novelle von Wieland bis Musil. Tübingen: Niemeyer.

Ralph, Marko (2006): Das Modell der „Wiedergeburt“ in ausgewählten Erzähltexten der fantastischen Literatur der Frühen Moderne. Kiel: GRIN Publishing.

Rassmann, Friedrich (1823): Pantheon deutscher, jetzt lebender Dichter und in die Belletristik eingreifender Schriftsteller; begleitet mit kurzen biografischen Notizen und der wichtigsten Literatur. Helmstedt: E. G. Fleckeisensche Buchhandlung.

Riedel, Wolfgang (2002): Weltgeschichte ein erhabenes Objekt. Zur Modernität von Schillers Geschichtsdenken. In: Peter Andre Alt u. a. (Hgg.): Prägnanter Moment. Festschrift für Hans-Jürgen Schings. Studien zur deutschen Literatur der Aufklärung und Klassik. Würzburg: Königshausen \& Neumann, 193-215.

Rosenberg, Rainer (2013): Verhandlungen des Literaturbegriffs. Studien zur Geschichte und Theorie der Literaturwissenschaft. Berlin: Akademie Verlag.

Rudolph, Andrea (2018): Modernitätsverhandlungen in schlesischen Novellen um 1830. Ästhetische, kulturelle und politische Dimensionen. Dettelsbach b. Würzburg: Dr. Röll.

Scävola, Emerentius (1832): Camaraobscura-Bilder. Bd. 1. Berlin: Schlesingsche Buch- und Musikhandlung, 1-105. 
Schlosser, Johann Georg (1781): Ueber die Seelenwanderung. Basel: C. A. Serini.

Schmidt, Andreas Gottfried (1840): Gallerie deutscher pseudonymer Schriftsteller vorzüglich des letzten Jahrzehnts. Ein Beitrag zur neuesten Literaturgeschichte. Grimma: Verlag des Verlags-Comptoirs.

Sengle, Friedrich (1972): Biedermeierzeit. Deutsche Literatur im Spannungsfeld zwischen Restauration und Revolution 1815-1848, 3 Bde. Bd. 2: Biedermeierzeit: Die Formenwelt. Stuttgart: Metzler.

Windischmann, Karl Joseph Hieronymus (Hg.) (1846): Friedrich Schlegel's Philosophische Vorlesungen aus dem Jahre 1804-1806. Nebst Fragmenten vorzüglich philosophisch-theologischen Inhalts, Dritter Theil, enthaltend den zweiten Band der ersten Abteilung, zweite Ausgabe. Bonn: Weber.

Zander, Helmut (1999): Geschichte der Seelenwanderung in Europa. Alternative religiöse Traditionen von der Antike bis heute. Darmstadt: Primus-Verlag. 